\title{
Gravity Lamp
}

\author{
Abhijeetsinh v Makwana ${ }^{1}$, Dhruv patel ${ }^{2}$, Darshan patel $^{3}$, Henil patel ${ }^{4}$, Akhil \\ patel $^{5}$, Assistant Professor ${ }^{1}$, UG Scholar ${ }^{2,3,4,5}$ \\ ${ }_{1,2,3,4,5}$ Department of Mechanical Engineering, Silver Oak College of Engineering \& Technology, GTU \\ Ahmedabad, Gujarat - India
}

\begin{abstract}
In recent times due to effects of pollution and global warming there is a need for generating power from renewable sources. The reason for generating power using gravity is that it is available all over the Earth, abundant and consistent too. In this project we designed a methodology wherein gravitational energy is further amplified in terms of its magnitude by using perpetual motion mechanism and hence can be successfully transformed into usable electrical energy. The basic concept of a gravity power generating mechanism is simple. Keywords: gravity power generating mechanism
\end{abstract}

\section{INTRODUCTION}

Cases of apparent perpetual motion can exist in nature, but either is not truly perpetual or cannot be used to do work without changing the nature of the motion. For example, the motion of a planet around its star may appear "perpetual," but interplanetary space is not completely frictionless, so planets' orbital motion is very gradually slowed over time. The flyby of a space probe past a planet can be used to speed up the probe but in doing so alters the motion and reduces the energy of the planet in its orbit around the Sun. The flow of current in a superconducting loop can be used as an energy storage medium, but just as with a battery, using it to power a device will remove the equivalent amount of energy from the current in the loop.

Despite the fact that successful isolated system perpetual motion devices are physically impossible in terms of the current understanding of the laws of physics, the pursuit of perpetualmotion remains popular. There are many ways to convert gravitational energy into electrical energy. Gravity lamp is one of the mechanisms.

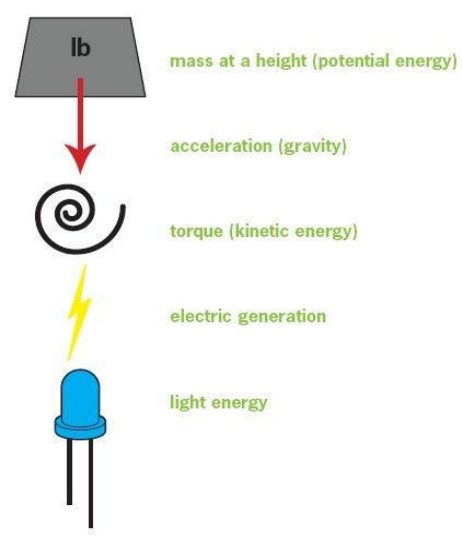

\section{METHODOLOGY}

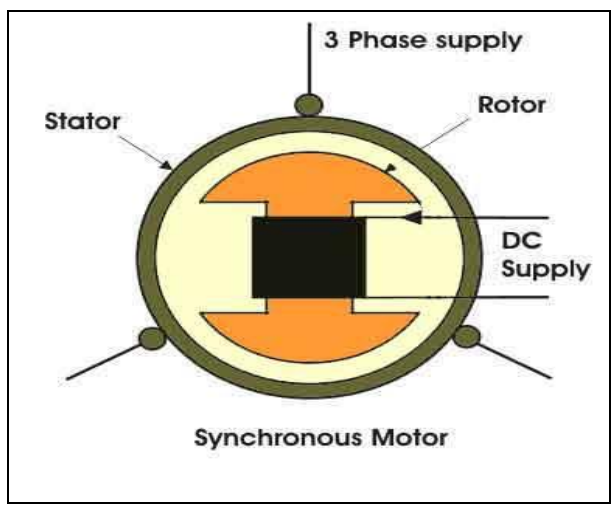

Main Features:

1) Synchronous motors are inherently not self starting. They require some external means to

2 b bring their speed close to synchronous speed to before they are synchronized.

3) The speed of operation of is in synchronism with the supply frequency and hence for

4 constant supply frequency they behave as constant speed motor irrespective of load

5) condition

6) This motor has the unique characteristics of operating under any electrical power factor.

7\} This makes it being used in electrical power factor improvement.

\section{PRINCIPLE}

Synchronous motor is a doubly excited machine i.e two electrical inputs are provided to it. It's stator winding which consists of a 3 phase winding is provided with 3 phase supply and rotor is provided with DC supply. The 3 phase stator winding carrying 3 phase currents produces 3 phase rotating magnetic flux. The rotor carrying DC supply also produces a constant flux. Considering the frequency 
to be $50 \mathrm{~Hz}$, from the above relation we can see that the 3 phase rotating flux rotates about 3000 revolution in $1 \mathrm{~min}$ or 50 revolutions in $1 \mathrm{sec}$. At a particular instant rotor and stator poles might be of same polarity (N-N or $\mathrm{S}-\mathrm{S})$ causing repulsive force on rotor and the very next second it will be N-S causing attractive force. But due to inertia of the rotor, it is unable to rotate in any direction due to attractive or repulsive force and remain in standstill condition. Hence it is not self starting. To overcome this inertia, rotor is initially fed some mechanical input which rotates it in same direction as magnetic field to a speed very close to synchronous speed. After some time magnetic locking occurs and the synchronous motor rotates in synchronism with the frequency.

\section{Application}

1) Synchronous motor having no load connected to its shaft is used for power factor improvement. Owing to its characteristics to behave at any electrical power factor, it is used in power system in situations where static capacitors are expensive.

2) Synchronous motor finds application where operating speed is less (around 500rpm) and high power is required. For power requirement from $35 \mathrm{~kW}$ to $2500 \mathrm{KW}$, the size, weight and cost of the corresponding three phase induction motor is very high. Hence these motors are preferably used. Ex- Reciprocating pump,compressor, rolling mills etc.

\section{Mild Steel Shaft:}

BS970 1983 on, PD970 2005 080A15, 080M15, $070 \mathrm{M} 20$.

BS970 1955 EN3A, EN3B, EN32B.

BSEN10083 C22, BS EN10084 C15/C16.

Werkstoff $1.0401,1.0426,1.0402,1.1140,1.1141$, $1.1148,1.1149,1.11511 .1208$.

Related grades SAE 1015/1017/1020/1023.

General purpose steel bars for machining, suitable for lightly stressed components including studs, bolts, gears and shafts. Often specified where weldability is a requirement. Can be casehardened to improve wear resistance. Available in bright rounds, squares and flats, and hot rolled rounds. Can be supplied in sawn blanks, and bespoke size blocks.

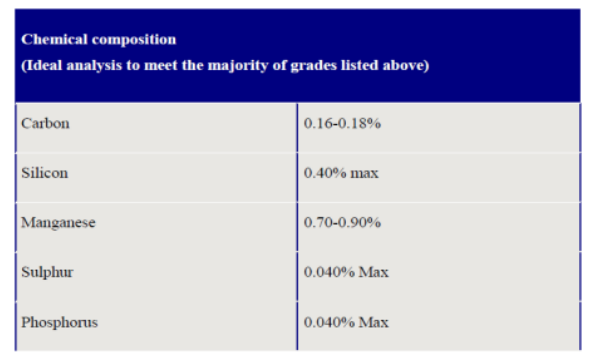

Table 3.1. Chemical Composition Of Mild Steel Shaft

\section{WORKING}

As shown in the diagram below, the whole idea is to have the masses fall as slowly as possible, taking as long as possible, while still causing the generator to turn fast enough to power the LED light. With the system below, even though the small sprocket is turning slowly, the outer edge of the large pulley/wheel that it's attached to is turning fast.

The generator we are using is a motor from a microwave oven, the one that slowly turns the tray inside the oven. If you turn the shaft of a motor manually then the motor acts as a generator and produces power. This motor has a lot of gears inside it, which make it such that turning the motor shaft slowly results in the magnets inside turning very fast, producing a useful amount of power.

It's in the use of the pulley system below, along with that particular motor as a generator, that the conversion from slow to fast is done.

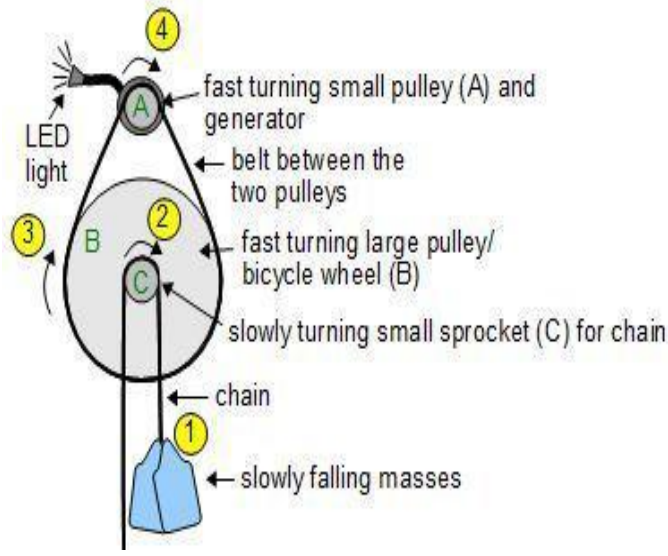

Fig.5.1 Working

\section{EXPERIMENTAL RESULT POWER OUTPUT}

$>$ So let us assume that we hang the Gravity light from a 1.5 meter height, and on high brightness it runs for 15-25 minutes.

$>$ The mass of the main bag is $8-16 \mathrm{~kg}$.

$>$ We know that the gravitational field strength of earth is $9.8 \mathrm{~N} / \mathrm{kg}$.

$>$ It can generate light around three watts LED bulb.

7.2 CALCULATIONS:
\begin{tabular}{|c|c|c|c|}
\hline SR NO. & GEAR & WEIGHT(kg) & TIME(min) \\
\hline 1 & $1^{\text {gt }}$ & 8 & 15 \\
\hline 2 & $2^{\text {td }}$ & 10 & 17.5 \\
\hline 3 & $3^{\text {td }}$ & 12 & 20 \\
\hline 4 & $4^{\text {th }}$ & 14 & 22.5 \\
\hline 5 & $5^{\text {th }}$ & 16 & 25 \\
\hline
\end{tabular}




\section{CONCLUSION}

When compared to other sources of energy like hydal, thermal, tidal, wind, nuclear etc. Gravity is more abundant and available everywhere on the earth. Moreover it is eco-friendly. Gravity lamp has been developed to provide clean, reliable and safe light, enabling people to break free from the economic, health and environmental hazards of kerosene lamps.

\section{REFRENECES}

[1] www.gravitylight.org

[2] www.indiegogo.com/projects/gravitylight

[3] en.m.wikipedia.org/wiki/Gravitylight 